\title{
DIAGNOSIS OF CHYTRIDIOMYCOSIS IN AMPHIBIANS BY HISTOLOGIC EXAMINATION
}

\author{
Lee Berger ${ }^{1,2}$, Rick Speare ${ }^{2}$ and Andrew Kent ${ }^{2}$ \\ ${ }^{1}$ CSIRO Australian Animal Health Laboratory, Ryrie Street, Geelong, Victoria 3220, Australia \\ ${ }^{2}$ School of Public Health and Tropical Medicine, James Cook University, Townsville, Queensland 4811, Australia \\ E-mail:lee.berger@dah.csiro.au; richard.speare@jcu.edu.au; andrew.kent@jcu.edu.au
}

\begin{abstract}
Chytridiomycosis is a fatal disease of post-metamorphic frogs and can be carried by healthy tadpoles. Here we describe methods for preparing and interpreting histologic sections from adults and tadpoles for the diagnosis of chytridiomycosis.
\end{abstract}

\section{Key words}

Chytrid fungus, chytridiomycosis, Batrachochytrium dendrobatidis, frogs

\section{Introduction}

A newly identified chytrid fungus in the genus Batrachochytrium (Longcore et al., 1999) that infects amphibians and can cause death has been found in Australia, South America, Central America and the USA (Berger et al., 1998; Pessier et al., 1999; Daszak et al., 1999). The chytrid fungus is the most common cause of death in Australian frogs (Berger et al., 1999) and has also been found in a small proportion of apparently healthy frogs and tadpoles (Berger et al., 1999).

The chytrid can be diagnosed by routine histology of skin specimens preserved in formalin or ethanol. Examination of unstained skin scrapings is a quick method, but requires greater expertise in identifying organisms. Chytrid culture from fresh specimens requires specialised methods (Longcore et al., 1999) and is difficult; so most diagnoses are made using histology.

Diagnosis using histology requires skills in microscopy, a knowledge of the morphology of the chytrid, a knowledge of normal histology of the skin, and an ability to differentiate the chytrid from other structures in the skin. The histology of the normal amphibian skin has been described by Patt and Patt (1970) and Green (1999). Pessier et al. (1999) describe the histological appearance of the amphibian chytrid, but this paper provides more detailed criteria so investigators unfamiliar with histopathology can make a diagnosis of chytrid infection. This technique may be used for surveying toe clips from wild and

http://www.jcu.edu.au/school/phtm/PHTM/frogs/histo/chhisto.htm Seen 1 December 1999. captive amphibians, surveying archived specimens, to test animals before translocation, and to determine the cause of mortality in the wild and in captivity so that appropriate management can be implemented.

Using histological techniques the chytrid fungus has been found in 31 species of amphibians in Australia, 10 species in USA, seven species in Panama and three species in Ecuador (Berger et al., 1999; Nichols et al., 1998; Carey et al., 1999). In Australia the technique has been used to start mapping the distribution of the chytrid and has identified three geographic foci: east coast, Adelaide and environs and southwest Western Australia (Berger et al., 1999).

Healthy tadpoles can carry chytrids for months. Tadpole skin does not become infected as it is not keratinised, but chytrids may infect the keratinised mouthparts. Healthy infected tadpoles may be highly prevalent in a population and so sampling tadpoles is a sensitive way of assessing a location. This paper refers to diagnosis in post metamorphic amphibians except where tadpoles are specifically mentioned.

\section{Methods}

The following description is based on histological sections prepared from tissue preserved in 10 per cent formalin or 70 per cent ethanol, embedded in paraffin, sectioned at $5 \mu \mathrm{m}$., and stained with haematoxylin and eosin (Drury \& Wallington, 1980).

Strips of skin from the pelvic region were embedded to obtain the maximum length of stratum corneum from the specimen 


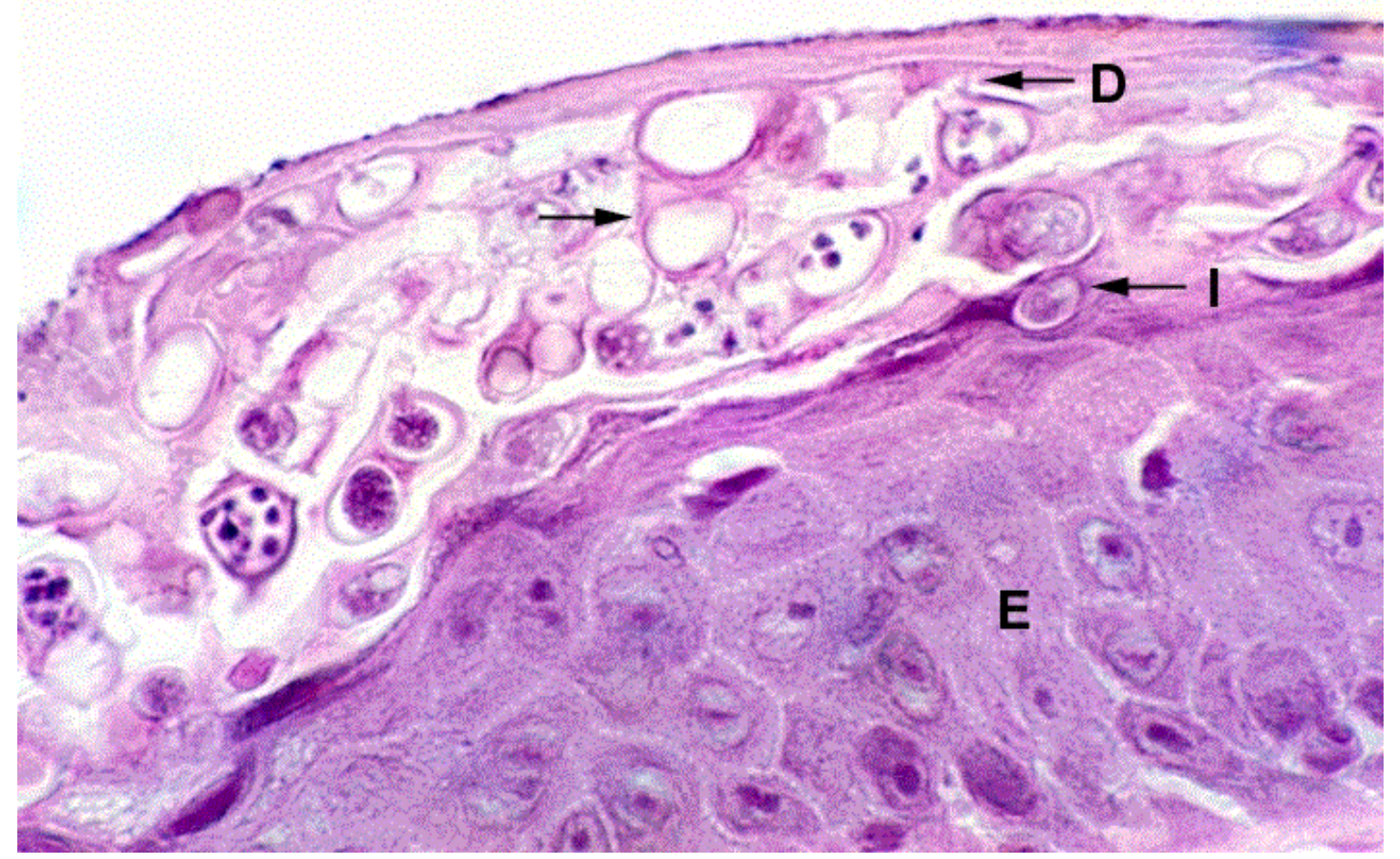

Figure 1. Section of skin from a heavily infected adult of Litoria caerulea. Note homogenous immature stage (I), zoosporangium with discharge papillae (D) containing zoospores, and empty zoosporangium formed after zoospores have discharged (arrow). $E=$ Epidermis.

available with the best plane for interpretation being a vertical section through the skin. Digits were examined by 1) sectioning a whole foot ventral side down or 2) sectioning a single toe (when testing live animals or when minimal mutilation of the carcass is required). For toes the maximum length of stratum corneum was obtained from a longitudinal section rather than a cross section. Larger digits, for example from amphibians with a snout vent length greater than $60 \mu \mathrm{m}$., were degloved by removing skin from the underlying phalanx and sectioning the skin without bone. Smaller toes, for example, from frogs with a snout vent length lesser than $50 \mu \mathrm{m}$., were usually sectioned without decalcification. Decalcification was required for larger toes if bone was not removed. Decalcification was performed by placing toes in EDTA for 48 hours at $37^{\circ} \mathrm{C}$ or in 10 per cent formic acid for three to five days prior to processing. For population surveys, or for smaller specimens about three serial sections were usually placed on the same slide to increase the length of stratum corneum available for examination. To prevent loss of toes during processing, sponge biopsy pads (Edward Keller, Hallam, Victoria) were used within cassettes. Skin was obtained from naturally and experimentally infected frogs and toads from Australia. Measurements were made with a calibrated eyepiece graticule.

\section{Specimens}

The chytrid fungus only invades the stratum corneum and stratum granulosum, in particular the subsurface layer (Fig. 1).
Therefore, skin is the only organ required for diagnosis. Since the keratinised surface layer of infected epidermis may slough and it contains organisms, sloughed epithelium can also be used for diagnosis.

\section{Site}

The fungus is not evenly distributed on the surface of the body. It is found most often in stratum corneum of the digits, then the ventral surface (especially the thighs and inguinal region) and less often on the dorsal surface (Pessier et al., 1999; Berger et al., 1998). In severe infections chytrids may be detected on the dorsal surface. In light infections the digits are the site of choice and have been used to conduct prevalence surveys of clinically normal frogs. Toe clips can be retained for examination and the amphibians released.

\section{Tadpoles}

Diagnosis in tadpoles can only be done by sacrificing the animal. Bathing in MS-222 (tricaine methanesulphonate, Ruth Consolidated Industries, Annandale) is a suitable method for euthanasia. A section through the mouthparts which includes the dark brown keratinised "teeth" is required for diagnosis. Large tadpoles are cut longitudinally down the midline with a scalpel and embedded with the cut surface downwards. Small tadpoles are best embedded whole on their side in the paraffin block, and then serially sectioned to reach the mouthparts. The 
size of the mouth varies between species, which affects the ease of obtaining a suitable section.

\section{Morphology of Batrachochytrium dendrobatidis \\ Shape}

In the stratum corneum the chytrid is roughly spherical with a discharge papilla projecting from the surface (Fig. 1), a good analogy being an inflated balloon with the neck of the balloon representing the discharge papilla. This overall structure is called a zoosporangium (Longcore et al., 1999). Occassional zoosporangia have several discharge papilla. Each zoosporangium appears to be intracellular. The discharge papilla

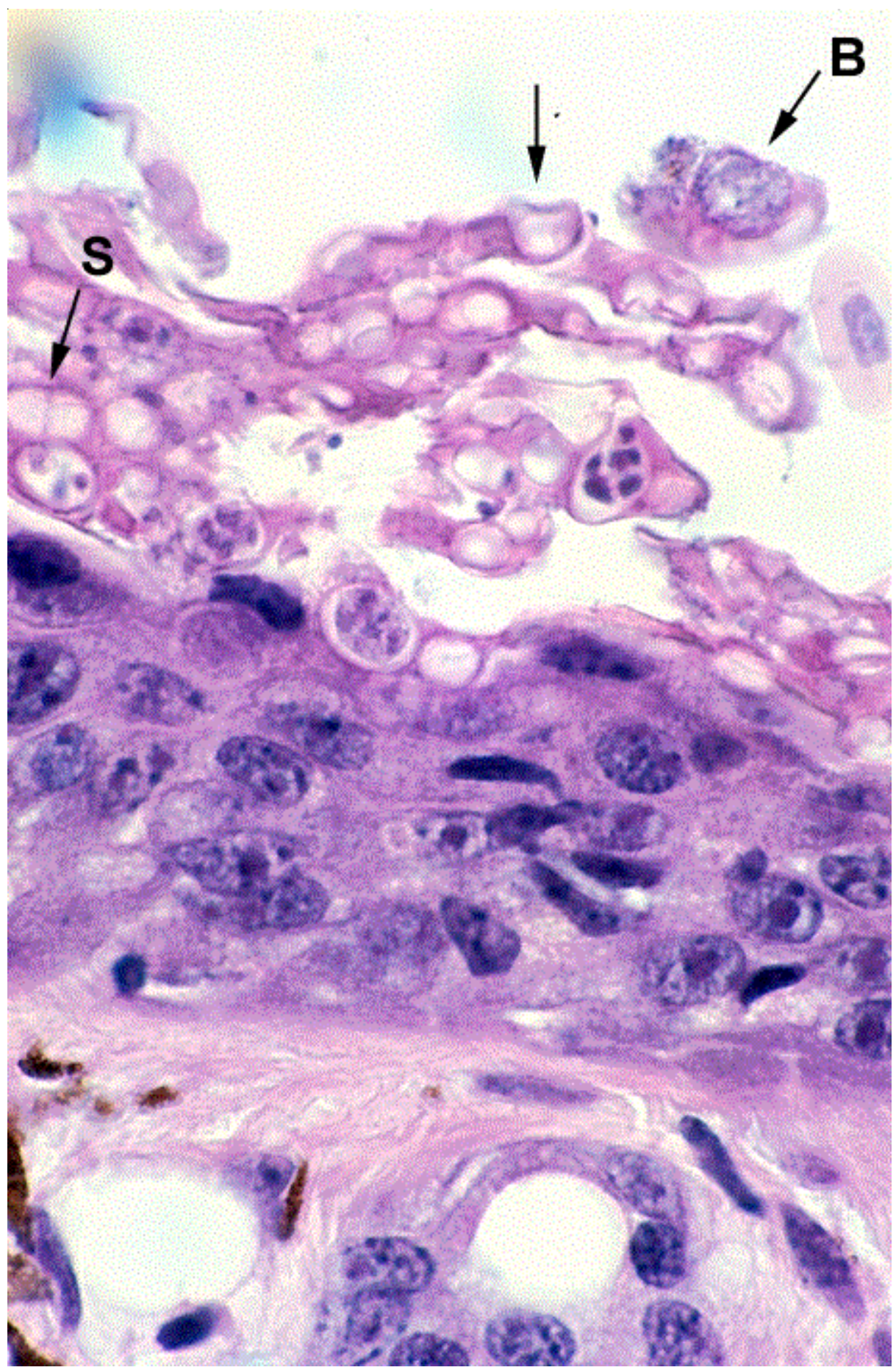

Figure 2. Section of skin from a Mixophyes fasciolatus with mostly empty sporangia present. Note empty collapsing sporangium (arrow) and one containing bacteria (B). One sporangium is divided by an internal septum (S). 

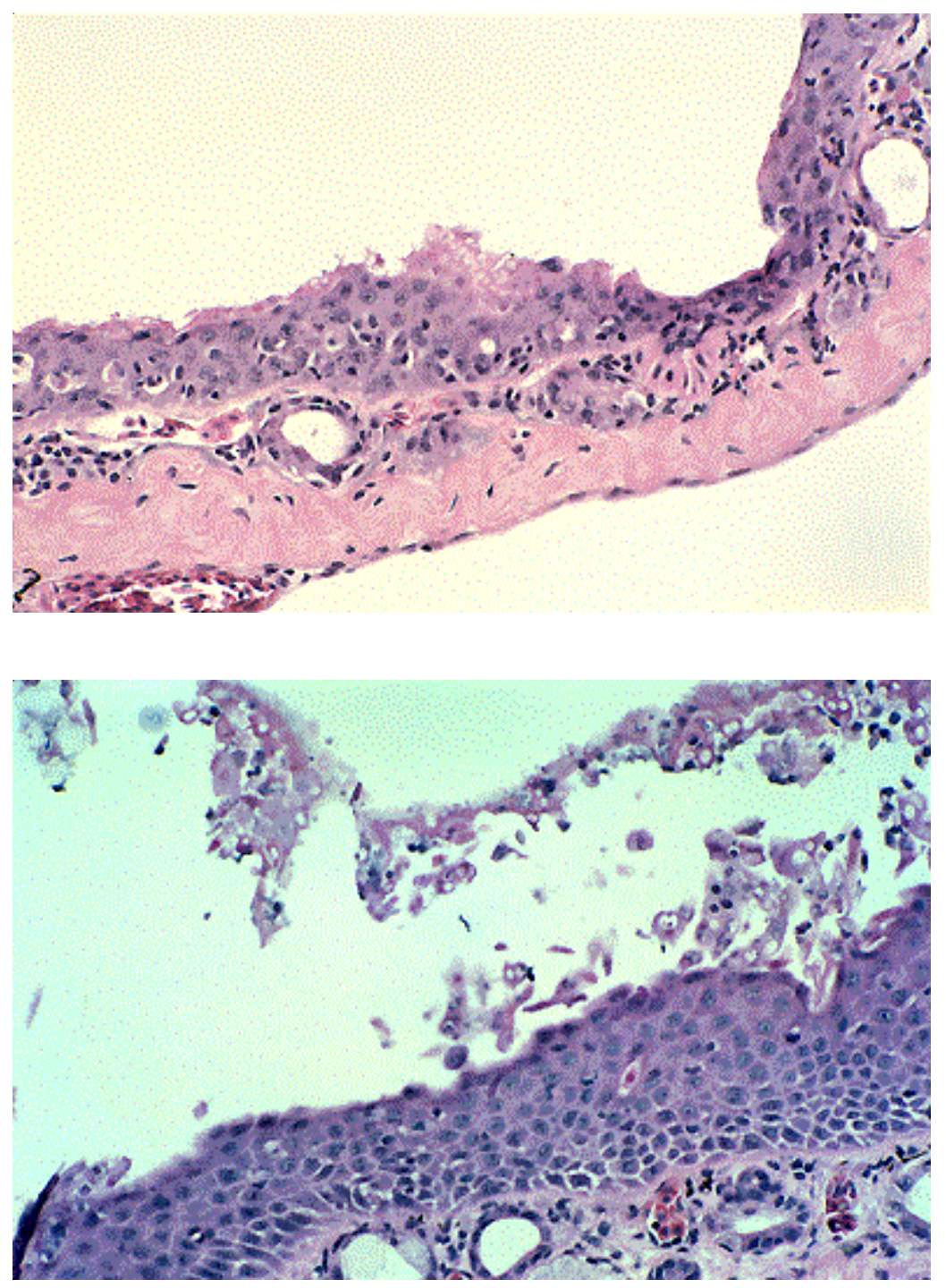

Figure 3. Section of skin from lightly infected Mixophyes fasciolatus with focal hyperkeratosis. The chytrid appears as small, clear circular spaces in the surface of the thickened epidermis.

Figure 4. Section of skin from metamorph of Mixophyes fasciolatus with the heavily infected stratum corneum sloughing, leaving few organisms on the epidermis in the left half of the image. The chytrid appears as empty spaces or dark circles in the sloughing epithelium.

projects beyond the surface epithelium and has a plug at the mouth (Berger et al., 1998). Discharge papillae can be seen in histological sections, but they are not common. Zoospores that develop in the zoosporangium escape through the open discharge tube.

\section{Wall}

The wall of the zoosporangium is smooth on outer and inner surfaces and very uniform in thickness. Its staining characteristics vary from eosinophilic to slightly basophilic or refractile. Variable amounts of keratin may closely surround the organism.

\section{Contents}

The contents of the zoosporangia vary with the developmental stage of the chytrid (Figs. $1 \& 2$ ). On histological sections four stages can be identified. The earliest stage contains a central mass which is basophilic and roughly spherical or oval. The mass is fairly homogenous in staining characteristics (Fig. 1). The mass divides to form zoospores. Zoospores are basophilic and appear in cross section as round or oval bodies often with poorly defined margins (Fig. 1), usually numbering about four to 10 depending on the plane of section. Once the zoospores are released via the discharge papilla, the zoosporangium is empty and retains its spherical shape (Fig. 1). In some empty stages, thin septae may divide the zoosporangium into internal compartments (Longcore et al., 1999). The next stage is collapse of the empty zoosporangium into an irregular shape (Fig. 2). During this terminal stage the empty shell sometimes becomes colonised by bacteria, and these are seen in section as basophilic rods or cocci inside the "empty" zoosporangium (Fig. 2). Bacteria can be confused with zoospores, but zoospores are larger than bacteria and less numerous. In frogs in terminal stages of chytridiomycosis, large numbers of bacteria may be seen between layers of sloughing keratin. Empty zoosporangia are the most common stage present, particularly in the sloughing 
surface layer. Empty zoosporangia may be missed unless looked for carefully. When examining sections, a useful guide to detection is the presence of clear spaces in the surface epithelium. If these are seen, examination should be made at higher power including oil immersion.

\section{Size}

In histological sections the diameter of zoosporangia vary with the plane of section with identifiable sections through the edge of a zoospore being $6 \mathrm{~mm}$. while maximum diameters are roughly $15 \mu \mathrm{m}$. Discharge tubes have a diameter of $2 \mu \mathrm{m}$ and a variable length, usually between $2-4 \mathrm{~mm}$., but the plane of section is rarely exactly down the long axis of the discharge tube and the usual appearance is of an outpocketing of the zoosporangial wall. The developing mass in the early zoosporangium has a diameter of approximately 4-8 $\mathrm{m} \mathrm{m}$. Zoospores range in size from $1 \mu \mathrm{m}$. to $2 \mu \mathrm{m}$. As for the zoosporangium sizes in section vary with the plane of section.

Size can be a useful guide in distinguishing zoosporangia from other bodies in the stratum corneum since zoosporangia are usually less than $15 \mu \mathrm{m}$. in diameter.

\section{Stains}

Chytridiomycosis can be diagnosed using the routine $\mathrm{H} \& \mathrm{E}$ stains. Special fungal stains, such as Periodic Acid Schiff (PAS) or silver stains (Drury \& Wallington, 1980) may be used, but do not appear to offer any additional benefit, except perhaps for confirming infection in cases where a few indistinct stages are present. Experiments are planned to evaluate if special stains will improve the sensitivity of diagnosis particularly by people less familiar with the chytrid fungus.

Polyclonal antibodies to $B$. dendrobatidis have recently been produced by Berger and Hyatt for use in an immunoperoxidase stain to detect antigen of the chytrid in tissue sections. This stain will be useful in confirming the identity of doubtful structures.

Occasionally other fungi invade the superficial epidermis of amphibians, for example cutaneous mucormycosis (Taylor et al., 1999c). Most have thread-like hyphae, but in cross section the hyphae can often have a circular appearance, and if the number of planes of section are few, the inexperienced examiner may confuse hyphae with chytrid sporangia. If special fungal stains (PAS, silver stain) are used in these cases, the filamentous nature of the hyphae are much more apparent than with $H \& E$, and more oblique sections plus septae become obvious. Special fungal stains therefore have a role in improving the specificity of diagnosis of other fungi which may be confused with chytrids.

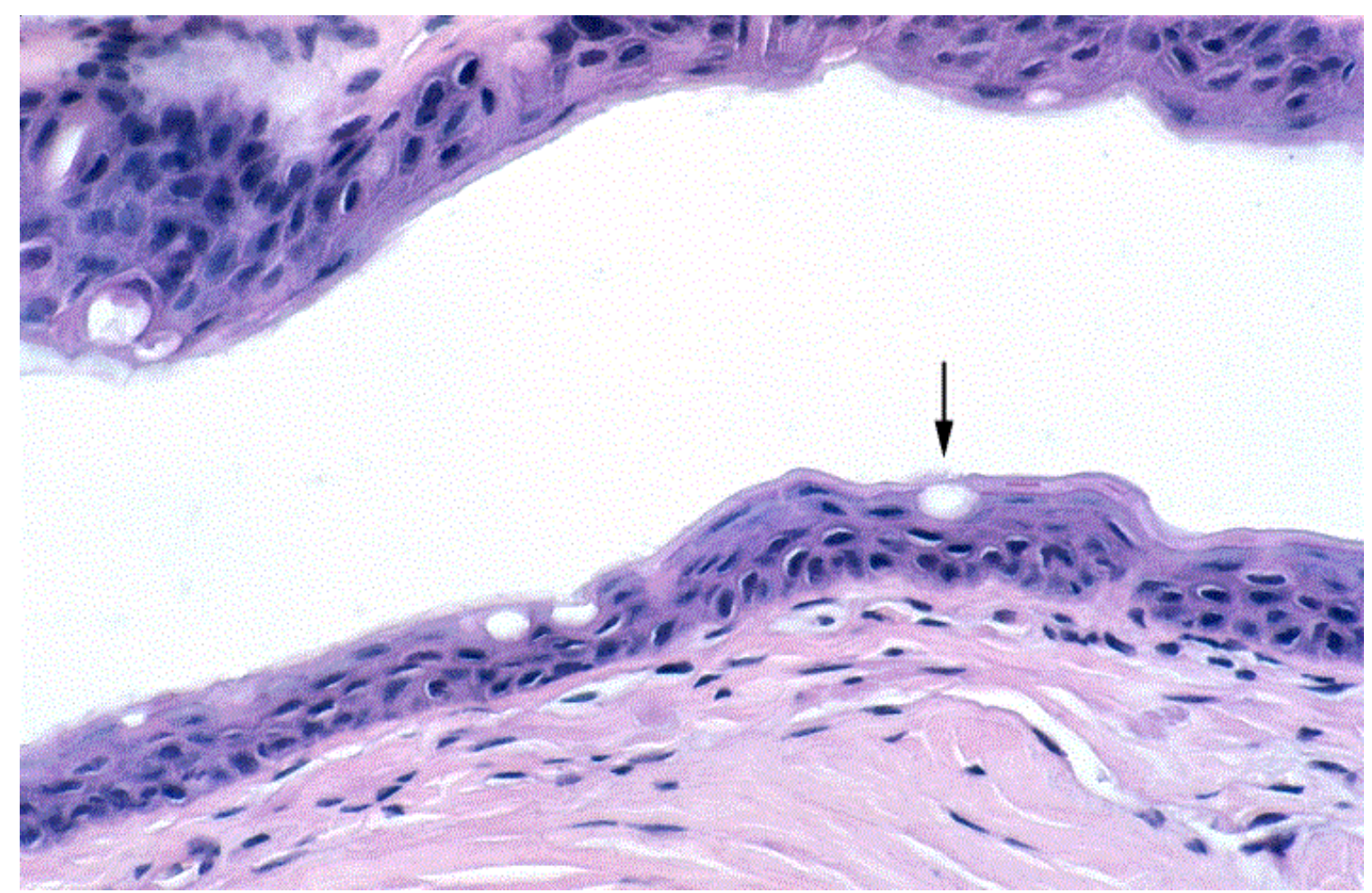

Figure 5. Normal toe skin from a Litoria chloris with vesicular structures (likely to be ducts from dermal glands) in the epidermis that appear similar to empty sporangia (arrow). Note that the clear spherical space lacks a definite cell wall and the adjacent epidermal cells are not hyperkeratotic. 


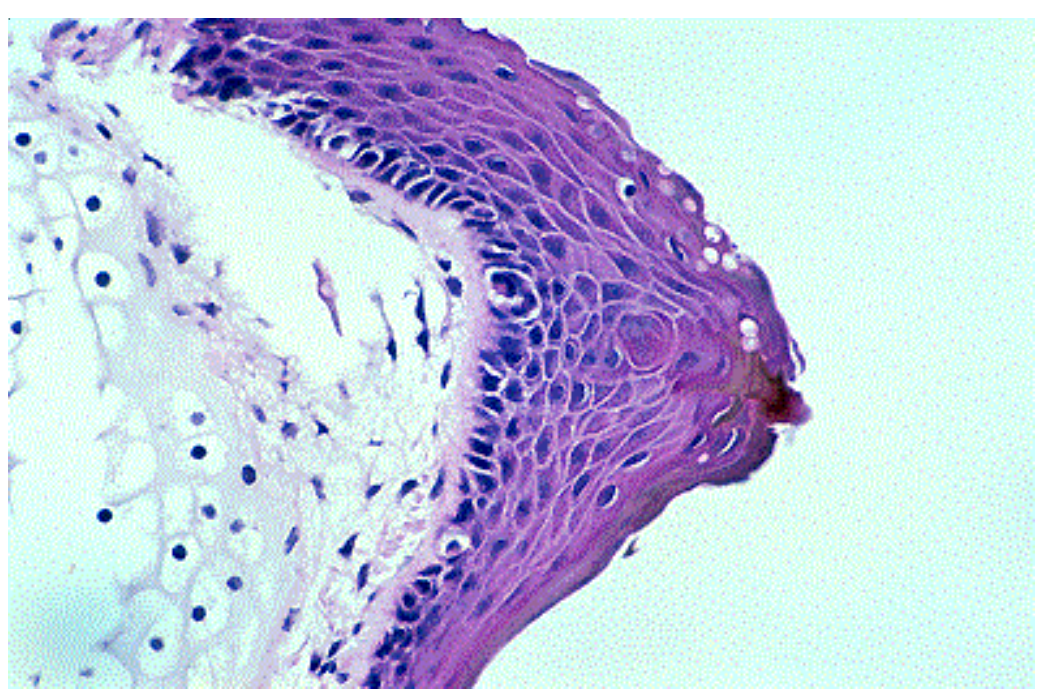

Figure 6. Section through mouthparts of a tadpole of Mixophyes fasciolatus with various stages present in the superficial keratinised epidermis.
In USA Basidiobolus ranarum has been reported to cause very similar superficial mycotic skin infections in Dwarf African Clawed Toads (Groff et al., 1991) and in Canadian toads (Taylor et al., 1999a, 1999b). However, there is controversy about whether these cases were due to Basidiobolus, or are misidentified cases of chytridiomycosis.

\section{Histopathology of chytridiomycosis}

In most cases the chytrid fungus is associated with focal hyperkeratosis and erosions in the area of stratum corneum adjacent to the organisms. Irregular thickening of the epidermis (hyperplasia) may be present as well as mild focal necrosis of epidermal cells. Vesicles may form between cells in the basal layer which coalesce and progress to ulcerations. There may be a negligible increase in inflammatory cells in the dermis. In light infections in clinically normal amphibians in which a single focus of organisms may be found, the hyperkeratosis is confined to that focus (Fig. 3), while in fatal infections hyperkeratosis is often widespread as are organisms. In some fatal cases there may be an extensive sloughing of the hyperkeratotic layer leaving an epidermis with few organisms (Fig. 4). However, in these cases chytrids can be detected in low numbers in the slightly keratinised surface layer, or chytrids may be seen in large numbers if the sloughed skin is examined. In cases where the skin has sloughed the diagnosis may be missed since the heavily infected surface layer has gone. With careful searching of the remaining epidermis chytrids can be found.

\section{Artifacts}

To consistently make a diagnosis of chytridiomycosis knowledge of the structures seen in normal skin is required. In addition there are a number of artifacts both in normal skin and in skin with other diseases that can be confused with chytrids. However, no other structure has exactly the same morphology, size, location and association with hyperkeratosis. Ducts from dermal glands often appear as spherical spaces between epidermal cells and may be confused with empty zoosporangia although they lack a distinct and complete wall, and are extracellular (Fig. 5). The basophilic immature stages of the chytrid in the subsurface layer can appear similar to epidermal cell nuclei, but are often surrounded by a clear halo. A PAS stain demonstrates the fungal wall around immature chytrids.

\section{Tadpoles}

The usual range of fungal stages may be present in tadpole mouthparts (Fig. 6). Tadpoles may have light infections accompanied by minimal pathology, or heavier infections with hyperkeratosis and occasional bacterial invasion.

\section{Technique of examination}

With experience epithelium can be scanned at x 200 power and suspicious organisms or areas on the epithelium easily detected. These suspicious areas should be examined at x 400 power to confirm the diagnosis. If one is unsure of the identity of a suspicious structure, examination under oil immersion (x1000) will usually improve the confidence of the decision. In heavy infections oil immersion is not required, but for light infections with few chytrids oil immersion may be required to make a definite diagnosis.

\section{Making a diagnosis}

We use the following steps to maximise the sensitivity of diagnosis of chytridiomycosis in histological sections.

1. Examine the stratum corneum or sloughed skin from the digits as first choice

2. Scan the stratum corneum at $\mathrm{x} 200$ or $\mathrm{x} 400$ power.

3. Look in the stratum corneum for

i) structures that have any of the morphological features described above and

ii) areas of hyperkeratosis or areas where the epidermal surface is irregular.

4. If these are found, examine using $x 400$ or $x 1000$ power. 
5. Make a diagnosis of Batrachochytrium on the basis of shape of zoosporangium (spherical, balloon shape, or collapsed sphere), nature of wall (thin, uniform, smooth outer and inner surfaces), type of contents (single central body, zoospores, bacteria), and diameter $(<15 \mu)$.

6. Differentiate from artifacts.

In most cases a diagnosis can be confidently made following the schema under points 1-5 above. Occasionally, however, for examiners with an inadequate level of knowledge of other structures occurring in the skin, the degree of confidence of the diagnosis may not be high. A diagnosis can be made easily if mature zoosporangia are seen, but is more difficult if only a few early or late stages are present. To confirm infection or to identify hyphal fungi use fungal stains such as PAS or silver stains, examine additional histological sections, or refer the slide to an expert. In screening amphibians for chytridiomycosis we classify results into negative (no chytrids found), confirmed positive (chytrids found), and suspect positive (possible chytrids found, but confirmation needed).

\section{Confirmation of Chytridiomycosis}

Currently, confirming that the structures seen are $B$. dendrobatidis relies on the level of expertise of the examiner. If a person is unsure of the identity, histological slides can be submitted to Lee Berger at AAHL or Rick Speare or Diana Mendez (diana.mendez@jcu.edu.au) at James Cook University. However, a polyclonal antibody against $B$. dendrobatidis has recently been developed by Lee Berger and Alex Hyatt at AAHL and early tests on histological slides show good specificity. Use of the polyclonal antibody to detect antigens of $B$. dendrobatidis offers promise of increasing the specificity and sensitivity of diagnoses, and making the identification less operator dependent. Suspect specimens submitted to AAHL can be tested using the polyclonal antibody. Other potential techniques include monoclonal antibodies and PCR-related DNA detection tests. Work is proceeding on these, but has not yet reached the stage of a useable diagnostic test.

\section{Acknowledgements}

Thanks to Frank Filippi for photography, Gerry Marantelli for supplying frogs and Megan Braun and Gail Russell for excellent histology.

\section{References}

Berger, L., R. Speare, P. Daszak, D.E. Green, A.A. Cunningham, C.L. Goggin, R. Slocombe, M.A. Ragan, A.D. Hyatt, K.R. McDonald, H.B. Hines, K.R. Lips, G. Marantelli and H. Parkes (1998). Chytridiomycosis causes amphibian mortality associated with population declines in the rain forests of Australia and Central America. Proceedings of the National Academy of Science, USA 95: 9031-9036. Berger. L., R. Speare and A. Hyatt (1999). Chytrid fungi and amphibian declines: Overview, implications and future directions. Pp. 21-31 In: Declines and Disappearances of Australian Frogs. A. Campbell (Ed). Environment Australia, Canberra.
Carey, C., N. Cohen and L. Rollins-Smith (1999). Amphibian declines: an immunological perspective. Developmental and Comparative Immunology 23: 459-472.

Daszak, P., L. Berger, A.A. Cunningham, A. Hyatt, D.E. Green and R. Speare (1999). Emerging infectious diseases and amphibian population declines. Emerging Infectious Diseases 5(5).

Drury, R.B. and E.A. Wallington (1980). Carleton's Histological Technique. Oxford University Press, 520 pp.

Green, D.E. (1999). Amphibian Medicine and Husbandry. Krieger Publishing.

Groff, J.M., A. Mughannam, T.S. McDowell, A. Wong, M.J. Dykstra, F.L. Frye and R.P. Hedrick (1991). An epizootic of cutaneous zygomycosis in cultured Dwarf African Clawed Frogs (Hymenochirus curtipes) due to Basidiobolus ranarum. J. of Medical and Veterinary Mycology, 29: 215-223.

Longcore, J.E., A.P. Pessier and D.K. Nichols (1999). Batrachochytrium dendrobatidis gen. et sp. nov., a chytrid pathogenic to amphibians. Mycologia 91: 219-227.

Nichols, D.K., A.P. Pessier and J.E. Longcore (1998). Cutaneous chytridiomycosis: an emerging disease? Proceedings of the American Association of Zoo Veterinarians pp. 269-271.

Patt, D.I. and G.R. Patt (1969). Comparative Vertebrate Histology. Harper and Row, New York p117-121

Pessier, A.P., D.K. Nichols, J.E. Longcore and M.S. Fuller (1999). Cutaneous chytridiomycosis in Poison Dart Frogs (Dendrobates spp.) and White's Tree Frogs (Litoria caerulea). J. of Veterinary Diagnostic Investigation 11: 194-199.

Taylor, S.K., E.S. Williams and K.W. Mills (1999a). Experimental exposure of Canadian toads to Basidiobolus ranarum. J. of Wildlife Diseases 35: 58-63.

Taylor, S.K., E.S. Williams and K.W. Mills (1999b). Mortality of captive Canadian toads from Basidiobolus ranarum mycotic dermatitis. J. of Wildlife Diseases 35: 64-69.

Taylor, S.K., E.S. Williams, A.C. Pier, K.W. Mills and M.D. Bock (1999c). Mucormycotic dermatitis in captive adult Wyoming Toads. J. of Wildlife Diseases 35: 70-74.

Editor's note: This document can also be viewed on the web (URL: http://www.jcu.edu.au/school/phtm/PHTM/ frogs/histo/chhisto.htm). This paper has been submitted by the authors to the Proceedings of the Australian Frog Symposium from who they are yet to hear. The paper is published for the first time in ZOOS' PRINT Journal, with the kind permission of the first author Dr. Lee Burger, to assist amphibian researchers in South Asia to be able to test for chytridiomycosis in amphibians in the region. There are no known cases of this infection occuring in amphibians in Asia. However, given its distribution in the USA, South America and Australia, it is likely that this may be occuring in South Asia but nothing is known because nobody has looked for it. A thorough study is required and it is hoped that this paper will help in such research within the country. 